\title{
On extensions of wavelet systems to dual pairs of frames
}

\author{
Ole Christensen*, Hong Oh Kim†, Rae Young Kim
}

October 30, 2018

\begin{abstract}
It is an open problem whether any pair of Bessel sequences with wavelet structure can be extended to a pair of dual frames by adding a pair of singly generated wavelet systems. We consider the particular case where the given wavelet systems are generated by the multiscale setup with trigonometric masks and provide a positive answer under extra assumptions. We also identify a number of conditions that are necessary for the extension to dual multiscale wavelet frames with any number of generators, and show that they imply that an extension with two pairs of wavelet systems is possible. Along the way we provide examples showing that extensions to dual frame pairs are attractive because they often allow better properties than the more popular extensions to tight frames.
\end{abstract}

Keywords: Bessel sequences, Dual frame pairs, Wavelet systems 2010 Mathematics Subject Classification: 42C15, 42C40

\section{Introduction}

Extension problems exist in a large variety in the frame literature. In its basic version the question is whether a given sequence of elements in a Hilbert space can be extended to a frame with prescribed properties. For example, it is natural to ask for extensions such that the resulting frame is computationally convenient, e.g., a tight frame or a frame for which a dual frame can be found easily. A natural generalization of this idea is to start with two sequences and ask for extension of these sequences to dual frame pairs.

It is known that any pair of Bessel sequences in a separable Hilbert space can be extended to a pair of dual frames by adding appropriate collections of vectors. But if we

${ }^{*}$ Department of Applied Mathematics and Computer Science, Technical University of Denmark, Building 303, 2800 Lyngby, Denmark (ochr@dtu.dk)

${ }^{\dagger}$ Department of Mathematical Sciences, KAIST, 373-1, Guseong-dong, Yuseong-gu, Daejeon, 305-701, Republic of Korea (kimhong@kaist.edu)

${ }^{\ddagger}$ Department of Mathematics, Yeungnam University, 214-1, Dae-dong, Gyeongsan-si, Gyeongsangbukdo, 712-749, Republic of Korea (rykim@ynu.ac.kr) 
require the added sequences to have a special structure or to satisfy certain constraints, many open problems appear. A key question is whether any given pair of Bessel sequences with wavelet structure can be extended to a pair of dual frames by adding a pair of wavelet systems, each with a single generator (see the paper [16] by D. Han for a discussion and conjecture about the corresponding tight case).

In this paper we will analyze this problem under the extra assumption that the given wavelet systems are generated by the MRA-setup as considered, e.g., in [18, 9, 12]. Given two scaling functions, we will consider the associated wavelet systems generated by letting the masks be trigonometric polynomials, and ask for extensions to dual pairs of frames by adding wavelet systems of the same type. We will first identify a condition on the refinement masks that is necessary for this extension to be possible, and then show that this condition is also sufficient for the possibility to extend to dual pairs using two generators. A stronger condition characterizes the possibility to extend the given wavelet systems to a pair of dual wavelet frames by adding a pair of wavelet systems, each with a single generator.

Note that Daubechies and B. Han already in [11] showed that for any given pair of scaling functions one can construct dual pairs of wavelet frames, each with two generators. Our setup is different from the one in [11]: in our extension of Bessel systems to dual pairs with two generators, we consider one of the pairs of Bessel sequences to be given, i.e., we only have freedom with respect to one pair of wavelet systems.

The rest of this introduction gives a short introduction to the key ingredients of the paper and connects our results to the literature. In Section 2 we present a few preliminary results on Bessel sequences and the mixed extension principle. The results about extensions with singly generated systems are stated in Section 3 , while the extension by systems with two generators is treated in Section 4 .

A sequence $\left\{f_{i}\right\}_{i \in I}$ in a separable Hilbert space $\mathcal{H}$ is called a frame if there exist constants $A, B>0$ such that

$$
A\|f\|^{2} \leq \sum_{i \in I}\left|\left\langle f, f_{i}\right\rangle\right|^{2} \leq B\|f\|^{2}, \forall f \in \mathcal{H}
$$

A frame is tight if we can choose $A=B$; and if at least the upper frame condition is satisfied, $\left\{f_{i}\right\}_{i \in I}$ is called a Bessel sequence. For any frame $\left\{f_{i}\right\}_{i \in I}$, there exist at least one dual frame, i.e., a frame $\left\{g_{i}\right\}_{i \in I}$ such that $f=\sum_{i \in I}\left\langle f, g_{k}\right\rangle f_{k}, \forall f \in \mathcal{H}$. A tight frame with $A=B=1$ leads to an expansion of arbitrary elements $f \in \mathcal{H}$ of exactly the same type as we know for orthonormal bases, i.e., $f=\sum_{i \in I}\left\langle f, f_{k}\right\rangle f_{k}, \forall f \in \mathcal{H}$. For more information on frames we refer to the books [10, 4].

In this paper we will exclusively consider systems of functions in $L^{2}(\mathbb{R})$ with wavelet structure, that is, collections of functions of the type $\left\{2^{j / 2} \psi\left(2^{j} x-k\right)\right\}_{j, k \in \mathbb{Z}}$ for a fixed function $\psi$. Considering the operators on $L^{2}(\mathbb{R})$ given by $T_{k} f(x):=f(x-k)$ and $D f(x):=$ $2^{1 / 2} f(2 x)$, the wavelet system can be written as $\left\{D^{j} T_{k} \psi\right\}_{j, k \in \mathbb{Z}}$.

Let $\mathbb{T}$ denote the unit circle which will be identified with $[-1 / 2,1 / 2]$. Also, for $f \in$ $L^{1}(\mathbb{R}) \cap L^{2}(\mathbb{R})$ we denote the Fourier transform by $\mathcal{F} f(\gamma)=\hat{f}(\gamma)=\int_{-\infty}^{\infty} f(x) e^{-2 \pi i x \gamma} d x$. As usual, the Fourier transform is extended to a unitary operator on $L^{2}(\mathbb{R})$.

In the entire paper we will use the following setup that appeared, e.g., in [12], except that we restrict our attention to trigonometric masks. 
General setup: Consider a function $\varphi \in L^{2}(\mathbb{R})$ such that

(i) $\widehat{\varphi}$ is continuous at the origin and $\hat{\varphi}(0)=1$;

(ii) There exists a 1-periodic trigonometric polynomial $m_{0}$ (called a refinement mask) such that

$$
\widehat{\varphi}(2 \gamma)=m_{0}(\gamma) \widehat{\varphi}(\gamma), \quad \text { a.e. } \gamma \in \mathbb{R}
$$

Given 1-periodic trigonometric polynomials $m_{1}, m_{2}, \ldots, m_{n}$, consider the functions $\psi_{\ell} \in$ $L^{2}(\mathbb{R})$ defined by

$$
\widehat{\psi}_{\ell}(2 \gamma)=m_{\ell}(\gamma) \widehat{\varphi}(\gamma), \ell=1, \ldots, n
$$

Note that the technical condition

$$
\sum_{k \in \mathbb{Z}}|\widehat{\varphi}(\gamma+k)|^{2} \leq K<\infty
$$

which is used in [12], automatically is satisfied in our setting. In fact, it is well known that $\varphi$ has compact support whenever the scaling equation (1.1) holds for a trigonometric polynomial $m_{0}$. Thus,

$$
\sum_{k \in \mathbb{Z}}|\widehat{\varphi}(\gamma+k)|^{2}=\sum_{k \in \mathbb{Z}}\langle\varphi, \varphi(\cdot-k)\rangle e^{-2 \pi i k \gamma}
$$

is a trigonometric polynomial.

We will base the analysis on the mixed extension principle (MEP) by Ron and Shen [18] which is formulated in terms of the $(n+1) \times 2$ matrix-valued functions $M$ and $\tilde{M}$ defined by

$$
M(\gamma)=\left(\begin{array}{cc}
m_{0}(\gamma) & m_{0}\left(\gamma+\frac{1}{2}\right) \\
m_{1}(\gamma) & m_{1}\left(\gamma+\frac{1}{2}\right) \\
\vdots & \vdots \\
m_{n}(\gamma) & m_{n}\left(\gamma+\frac{1}{2}\right)
\end{array}\right), \quad \widetilde{M}(\gamma)=\left(\begin{array}{cc}
\widetilde{m}_{0}(\gamma) & \widetilde{m}_{0}\left(\gamma+\frac{1}{2}\right) \\
\widetilde{m}_{1}(\gamma) & \widetilde{m}_{1}\left(\gamma+\frac{1}{2}\right) \\
\vdots & \vdots \\
\widetilde{m}_{n}(\gamma) & \widetilde{m}_{n}\left(\gamma+\frac{1}{2}\right)
\end{array}\right)
$$

Formulated for trigonometric masks, the MEP reads as follows:

Proposition 1.1 Assume that $\varphi, \widetilde{\varphi} \in L^{2}(\mathbb{R})$ satisfy the conditions in the general setup, with associated masks $m_{0}, \widetilde{m}_{0}$. For each $\ell=1, \cdots, n$, let $m_{\ell}, \widetilde{m}_{\ell}$ be trigonometric polynomials and define $\psi_{\ell}, \widetilde{\psi}_{\ell} \in L^{2}(\mathbb{R})$ by (1.2). Assume that $\left\{D^{j} T_{k} \psi_{i}\right\}_{i=1, \cdots, n ; j, k \in \mathbb{Z}}$ and $\left\{D^{j} T_{k} \widetilde{\psi}_{i}\right\}_{l=1, \cdots, n ; j, k \in \mathbb{Z}}$ are Bessel sequences. If the corresponding matrix-valued functions $M$ and $\widetilde{M}$ satisfy

$$
\widetilde{M}(\gamma)^{*} M(\gamma)=I, \quad \gamma \in \mathbb{T}
$$

then $\left\{D^{j} T_{k} \psi_{i}\right\}_{i=1, \cdots, n ; j, k \in \mathbb{Z}}$ and $\left\{D^{j} T_{k} \widetilde{\psi}_{i}\right\}_{l=1, \cdots, n ; j, k \in \mathbb{Z}}$ form dual frames for $L^{2}(\mathbb{R})$. 
The MEP was later extended to the mixed oblique extension principle in [9, 12]; these papers also contain several explicit examples. Other papers about the MEP include [1, 13, 15.

Most of the concrete wavelet frame constructions in the literature are obtained via the related unitary extension principle [18] and its variants, which lead to tight frame constructions (see, e.g., the papers [19, 17, 14, 3, 6], just to mention a few out of many). But it is already noted in, e.g., [12, 9] that the extra flexibility in the MEP frequently leads to more attractive constructions, a claim that is also supported by some of the results in the current paper. For example, we consider a case where the extension of a wavelet system to a tight frame introduces a wavelet generator without compact support, while the extension to a dual pair of frames is possible with compactly supported generators.

Note that the analysis in the current paper is complementary to the one in [5]. In [5] we formulated the general question whether any pair of Bessel sequences $\left\{D^{j} T_{k} \psi_{1}\right\}_{j, k \in \mathbb{Z}}$ and $\left\{D^{j} T_{k} \widetilde{\psi}_{1}\right\}_{j, k \in \mathbb{Z}}$ can be extended to a pair of dual frames by adding a wavelet system to each of the given Bessel sequences. A sufficient condition for a positive answer turned out to be that $\widehat{\psi_{1}}$ is compactly supported on $[-1,1]$. In contrast, the extension principle applied in the current paper involves functions that are compactly supported in time.

Finally, for the sake of the non-specialist, we note that it is known that the dual frames of a wavelet frame not necessarily have wavelet structure: there are cases where no dual wavelet frame exist at all (see, e.g., the books by Chui [7] and Daubechies [10]), and there are cases where some duals have wavelet structure and some do not (see the paper by Bownik and Weber [2]). This issue is one of the key motivations behind the various extension principles, which construct, simultaneously, a frame and a dual with wavelet structure.

\section{Preliminaries on Bessel sequences and the MEP}

In the entire paper we assume that we have given trigonometric polynomials $m_{0}, m_{1}, \widetilde{m}_{0}, \widetilde{m}_{1}$ as described in the general setup. We will search for trigonometric polynomials $m_{2}, \cdots, m_{n}$ and $\widetilde{m}_{2}, \cdots, \widetilde{m}_{n}$ for the cases $n=2$ and $n=3$ satisfying the condition (1.4), i.e.,

$$
\widetilde{M}(\gamma)^{*} M(\gamma)=I, \quad \gamma \in \mathbb{T}
$$

Note that (2.1) is equivalent to the two conditions

$$
\begin{aligned}
& \sum_{\ell=0}^{n} \overline{m_{\ell}(\gamma)} \widetilde{m}_{\ell}(\gamma)=1, \quad \gamma \in \mathbb{T}, \\
& \sum_{\ell=0}^{n} \overline{m_{\ell}(\gamma)} \widetilde{m}_{\ell}(\gamma+1 / 2)=0, \quad \gamma \in \mathbb{T} .
\end{aligned}
$$

In Proposition 1.1 we need that $\left\{D^{j} T_{k} \psi_{i}\right\}_{i=1, \cdots, n ; j, k \in \mathbb{Z}}$ and $\left\{D^{j} T_{k} \widetilde{\psi}_{i}\right\}_{l=1, \cdots, n ; j, k \in \mathbb{Z}}$ are Bessel sequences. Since these systems are finite union of wavelet systems, it is sufficient that each of these form a Bessel sequence. The following lemma provides necessary and sufficient conditions for this: 
Lemma 2.1 Let $\varphi \in L^{2}(\mathbb{R})$ be a scaling function, with a refinement mask $m_{0}$ satisfying the conditions in the general setup. Let $m$ be a trigonometric polynomial and define $\psi$ by

$$
\widehat{\psi}(2 \gamma)=m(\gamma) \widehat{\varphi}(\gamma), \ell=1, \ldots, n .
$$

Then the following are equivalent:

(a) $\left\{D^{j} T_{k} \psi\right\}_{j, k \in \mathbb{Z}}$ is a Bessel sequence;

(b) $m(0)=0$.

Proof. (a) $\Rightarrow$ (b) : Assume that $\left\{D^{j} T_{k} \psi\right\}_{j, k \in \mathbb{Z}}$ is a Bessel sequence with bound $B$. Using [8, Theorem 1] (or see Proposition 11.2.2 in [4]), we have $\sum_{j \in \mathbb{Z}}\left|\widehat{\psi}\left(2^{j} \gamma\right)\right|^{2} \leq B$. By the conditions in the general setup, $\widehat{\psi}$ is continuous at the origin, so it follows that $\widehat{\psi}(0)=0$. Hence, via (2.4) and the assumption $\widehat{\varphi}(0)=1$ we conclude that $m(0)=0$.

(b) $\Rightarrow$ (a) : See [11, Lemma 2.1].

Example 2.2 Let $B_{N}, N \in \mathbb{N}$, denote the $N$ th order B-spline, defined recursively by

$$
B_{1}:=\chi_{[0,1]}, B_{N+1}:=B_{1} * B_{N} .
$$

It is well known that $\varphi:=B_{N}$ satisfies the conditions in the general setup with $m_{0}(\gamma)=$ $\left(e^{-\pi i \gamma} \cos (\pi \gamma)\right)^{N}$. Thus Lemma 2.1 shows that $\left\{D^{j} T_{k} B_{N}\right\}_{j, k \in \mathbb{Z}}$ is not a Bessel sequence in $L^{2}(\mathbb{R})$. On the other hand, a finite linear combination

$$
\psi_{1}(x)=\sum_{k=-K}^{K} c_{k} B_{N}(2 x-k)
$$

generates a Bessel sequences $\left\{D^{j} T_{k} \psi_{1}\right\}_{j, k \in \mathbb{Z}}$ if and only if $\sum_{k=-K}^{K} c_{k}=0$.

The following example demonstrates that the matrix condition in Theorem 1.1 is not sufficient for duality, i.e., the assumption of the sequences $\left\{D^{j} T_{k} \psi_{i}\right\}_{i=1, \cdots, n ; j, k \in \mathbb{Z}}$ and $\left\{D^{j} T_{k} \widetilde{\psi}_{i}\right\}_{l=1, \cdots, n ; j, k \in \mathbb{Z}}$ being Bessel sequences is essential:

Example 2.3 Consider the scaling functions $\varphi(x)=\widetilde{\varphi}(x)=B_{2}(x)$ and

$$
\begin{aligned}
& \psi_{1}(x)=\widetilde{\psi}_{1}(x)=-\frac{1}{2} B_{2}(2 x)+B_{2}(2 x-1)-\frac{1}{2} B_{2}(2 x-2), \\
& \psi_{2}(x)=-\frac{1}{4} B_{2}(2 x+2)+\frac{1}{2} B_{2}(2 x)-\frac{1}{4} B_{2}(2 x-2), \quad \widetilde{\psi}_{2}(x)=2 B_{2}(2 x),
\end{aligned}
$$

with the associated masks

$$
\begin{aligned}
& m_{0}(\gamma)=\widetilde{m}_{0}(\gamma)=e^{-2 \pi i \gamma} \cos ^{2}(\pi \gamma), \\
& m_{1}(\gamma)=\widetilde{m}_{1}(\gamma)=e^{-2 \pi i \gamma} \sin ^{2}(\pi \gamma), \quad m_{2}(\gamma)=2 \cos ^{2}(\pi \gamma) \sin ^{2}(\pi \gamma), \quad \widetilde{m}_{2}(\gamma)=1 .
\end{aligned}
$$

Then the MEP-condition (1.4) with $n=2$ in Proposition 1.1 is satisfied. But by Lemma 2.1 we know that $\left\{D^{j} T_{k} \widetilde{\psi}_{2}\right\}_{j, k \in \mathbb{Z}}$ is not a Bessel sequence. Hence $\left\{D^{j} T_{k} \psi_{i}\right\}_{i=1,2 ; j, k \in \mathbb{Z}}$ and $\left\{D^{j} T_{k} \widetilde{\psi}_{i}\right\}_{l=1,2 ; j, k \in \mathbb{Z}}$ does not form dual frames for $L^{2}(\mathbb{R})$. 
In Lemma 2.5 we will state three necessary conditions for the existence of MEP-type

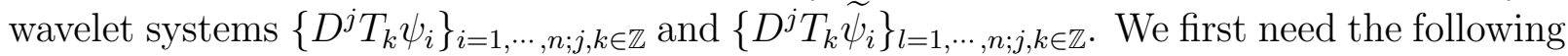
factorizations:

\section{Lemma 2.4}

(i) Let $f$ be a 1-periodic trigonometric polynomial with $f(0)=0$. Then $f(\gamma)=e^{-\pi i \gamma} \sin (\pi \gamma) \Lambda_{1}(\gamma)$ for a 1-periodic trigonometric polynomial $\Lambda_{1}$;

(ii) Let $g$ be a 1-periodic trigonometric polynomial with $g(1 / 2)=0$. Then $g(\gamma)=e^{-\pi i \gamma} \cos (\pi \gamma) \Lambda_{2}(\gamma)$ for a 1-periodic trigonometric polynomial $\Lambda_{2}$.

Proof. For the proof of (i), write $f$ as $f(\gamma)=\sum_{k \in \mathbb{Z}} c_{k} e^{-2 \pi i k \gamma}$. Since $f(0)=\sum_{k \in \mathbb{Z}} c_{k}=0$, we have $f(\gamma)=\sum_{k \neq 0} c_{k}\left(e^{-2 \pi i k \gamma}-1\right)$. Define $f_{+}$and $f_{-}$by

$$
f_{+}(\gamma):=\sum_{k \in \mathbb{N}} c_{k}\left(e^{-2 \pi i k \gamma}-1\right), \quad f_{-}(\gamma):=\sum_{k \in \mathbb{N}} c_{-k}\left(e^{2 \pi k i \gamma}-1\right)
$$

Then we see that

$$
\begin{aligned}
f_{+}(\gamma)=\sum_{k \in \mathbb{N}} c_{k}\left(e^{-2 \pi i \gamma}-1\right) \sum_{\ell=0}^{k-1} e^{-2 \pi i \ell \gamma} & =e^{-\pi i \gamma} \sin (\pi \gamma)\left(-2 i \sum_{k \in \mathbb{N}} c_{k} \sum_{\ell=0}^{k-1} e^{-2 \pi i \ell \gamma}\right) \\
=: & e^{-\pi i \gamma} \sin (\pi \gamma) \Lambda_{+}(\gamma) .
\end{aligned}
$$

Similarly,

$$
f_{-}(\gamma)=e^{-\pi i \gamma} \sin (\pi \gamma)\left(2 i \sum_{k \in \mathbb{N}} c_{-k} \sum_{\ell=1}^{k} e^{2 \pi i \ell \gamma}\right)=: e^{-\pi i \gamma} \sin (\pi \gamma) \Lambda_{-}(\gamma)
$$

Then we have $f(\gamma)=f_{+}(\gamma)+f_{-}(\gamma)=e^{-\pi i \gamma} \sin (\pi \gamma) \Lambda_{1}(\gamma)$, where $\Lambda_{1}(\gamma):=\Lambda_{+}(\gamma)+\Lambda_{-}(\gamma)$ is a 1-periodic trigonometric polynomial. This proves (i).

For the proof of (ii), let $\tilde{g}(\gamma):=g(\gamma+1 / 2)$. Since $\tilde{g}(0)=0$, there exists a 1 -periodic trigonometric polynomial $\Lambda$ such that $\tilde{g}(\gamma)=e^{-\pi i \gamma} \sin (\pi \gamma) \Lambda(\gamma)$. Then we have $g(\gamma)=$ $\tilde{g}(\gamma-1 / 2)=e^{-\pi i \gamma} \cos (\pi \gamma) \Lambda_{2}(\gamma)$, where $\Lambda_{2}(\gamma):=-i \Lambda(\gamma-1 / 2)$. This proves (ii).

Lemma 2.5 Under the hypothesis of Proposition 1.1, the following hold:

(a) $m_{\ell}(0)=\widetilde{m}_{\ell}(0)=0, \ell=1,2, \cdots, n$;

(b) $m_{0}(1 / 2)=\widetilde{m}_{0}(1 / 2)=0$;

(c) $1-\overline{m_{0}(\gamma)} \widetilde{m}_{0}(\gamma)=\sin ^{2}(\pi \gamma) \Lambda(\gamma)$ for some 1-periodic trigonometric polynomial $\Lambda$. 
Proof. (a) : This follows by Lemma 2.1.

(b) : Note that $m_{0}(0)=\widetilde{m}_{0}(0)=1$ by the assumptions in the general setup. This together with (a) and (2.3) imples $m_{0}(1 / 2)=\widetilde{m}_{0}(1 / 2)=0$.

(c) : By (a) and Lemma 2.4, the functions $m_{\ell}, \widetilde{m}_{\ell}, \ell=1,2 \cdots, n$, can be factorized as

$$
m_{\ell}(\gamma)=e^{-\pi i \gamma} \sin (\pi \gamma) \Lambda_{\ell}(\gamma), \widetilde{m}_{\ell}(\gamma)=e^{-\pi i \gamma} \sin (\pi \gamma) \widetilde{\Lambda}_{\ell}(\gamma),
$$

for some 1-periodic trigonometric polynomials $\Lambda_{\ell}, \widetilde{\Lambda}_{\ell}$. Combining this with (2.2) leads to $1-\overline{m_{0}(\gamma)} \widetilde{m}_{0}(\gamma)=\sin ^{2}(\pi \gamma) \Lambda(\gamma)$, where $\Lambda(\gamma)=\sum_{\ell=1}^{n} \bar{\Lambda}_{\ell}(\gamma) \widetilde{\Lambda}_{\ell}(\gamma)$.

In Section 4 we will show that, on the other hand, the assumptions (a), (b) and (c) in Lemma 2.5 implies that the wavelet systems $\left\{D^{j} T_{k} \psi_{1}\right\}_{j, k \in \mathbb{Z}},\left\{D^{j} T_{k} \widetilde{\psi_{1}}\right\}_{j, k \in \mathbb{Z}}$ can be extended to pairs of dual wavelet frames by adding two wavelet systems.

\section{$3 \quad$ Extension with one pair of generators}

In the rest of the paper we will consider scaling functions $\varphi, \widetilde{\varphi} \in L^{2}(\mathbb{R})$ as in the general setup, with associated trigonometric polynomial masks $m_{0}, \widetilde{m_{0}}$. Assuming that we have given trigonometric polynomials $m_{1}, \widetilde{m_{1}}$ and defined the associated functions $\psi_{1}, \widetilde{\psi_{1}}$ by (1.2), our goal is to extend the Bessel sequences $\left\{D^{j} T_{k} \psi_{1}\right\}_{j, k \in \mathbb{Z}},\left\{D^{j} T_{k} \widetilde{\psi_{1}}\right\}_{j, k \in \mathbb{Z}}$ to pairs of dual wavelet frames. It turns out to be convenient to consider the functions $\widetilde{M}_{\alpha}$ and $\widetilde{M}_{\beta}$, defined by

$$
\begin{aligned}
& \widetilde{M}_{\alpha}(\gamma):=1-\overline{m_{0}(\gamma)} \widetilde{m}_{0}(\gamma)-\overline{m_{1}(\gamma)} \widetilde{m}_{1}(\gamma) \\
& \widetilde{M}_{\beta}(\gamma):=-\overline{m_{0}(\gamma)} \widetilde{m}_{0}(\gamma+1 / 2)-\overline{m_{1}(\gamma)} \widetilde{m}_{1}(\gamma+1 / 2) .
\end{aligned}
$$

Lemma 3.1 If the conditions (a), (b) and (c) in Lemma 2.5 are satisfied, then $\widetilde{M}_{\alpha}$ and $\widetilde{M}_{\beta}$ can be factorized as

$$
\widetilde{M}_{\alpha}(\gamma)=\sin ^{2}(\pi \gamma) \Lambda_{\alpha}(\gamma), \quad \widetilde{M}_{\beta}(\gamma)=-i \sin (\pi \gamma) \cos (\pi \gamma) \Lambda_{\beta}(\gamma)
$$

for some 1-periodic trigonometric polynomials $\Lambda_{\alpha}$ and $\Lambda_{\beta}$.

Proof. By Lemma 2.4, and (a) and (b) of Lemma 2.5, $m_{\ell}, \tilde{m}_{\ell}, \ell=0,1$ can be factorized as

$$
\begin{array}{ll}
m_{0}(\gamma)=e^{-\pi i \gamma} \cos (\pi \gamma) \Lambda_{0}(\gamma), & \widetilde{m}_{0}(\gamma)=e^{-\pi i \gamma} \cos (\pi \gamma) \widetilde{\Lambda}_{0}(\gamma), \\
m_{1}(\gamma)=e^{-\pi i \gamma} \sin (\pi \gamma) \Lambda_{1}(\gamma), & \widetilde{m}_{1}(\gamma)=e^{-\pi i \gamma} \sin (\pi \gamma) \widetilde{\Lambda}_{1}(\gamma),
\end{array}
$$

for some 1-periodic trigonometric polynomials $\Lambda_{\ell}, \widetilde{\Lambda}_{\ell}$ for $\ell=0,1$. Together with (c) of Lemma 2.5 this implies

$$
\widetilde{M}_{\alpha}(\gamma)=\sin ^{2}(\pi \gamma)\left(\Lambda(\gamma)-\overline{\Lambda_{1}(\gamma)} \widetilde{\Lambda}_{1}(\gamma)\right)=: \sin ^{2}(\pi \gamma) \Lambda_{\alpha}(\gamma)
$$


and

$$
\begin{aligned}
\widetilde{M}_{\beta}(\gamma) & =-i \sin (\pi \gamma) \cos (\pi \gamma)\left(\overline{\Lambda_{0}(\gamma)} \widetilde{\Lambda}_{0}(\gamma+\pi / 2)-\overline{\Lambda_{1}(\gamma)} \widetilde{\Lambda}_{1}(\gamma+\pi / 2)\right) \\
& =:-i \sin (\pi \gamma) \cos (\pi \gamma) \Lambda_{\beta}(\gamma)
\end{aligned}
$$

as desired.

We are now ready to state a condition for extension of MRA-type wavelet systems $\left\{D^{j} T_{k} \psi_{1}\right\}_{j, k \in \mathbb{Z}},\left\{D^{j} T_{k} \widetilde{\psi}_{1}\right\}_{j, k \in \mathbb{Z}}$ to dual frames $\left\{D^{j} T_{k} \psi_{\ell}\right\}_{\ell=1,2 ; j, k \in \mathbb{Z}},\left\{D^{j} T_{k} \widetilde{\psi}_{\ell}\right\}_{\ell=1,2 ; j, k \in \mathbb{Z}}$. Note that in Theorem 3.2 below, the condition (i) in (I) means that $\left\{D^{j} T_{k} \psi_{2}\right\}_{j, k \in \mathbb{Z}}$ and $\left\{D^{j} T_{k} \widetilde{\psi}_{2}\right\}_{j, k \in \mathbb{Z}}$ are Bessel sequences, while (ii) simply is the MEP-condition. We also note that the proof shows how to choose the corresponding masks $m_{2}, \widetilde{m_{2}}$. With this information we can find the functions $\psi_{2}, \widetilde{\psi_{2}}$ explicitly: if, e.g., $m_{2}(\gamma)=\sum d_{k} e^{2 \pi i k \gamma}$, then $\psi_{2}=2 \sum d_{k} \varphi(2 x+k)$.

Theorem 3.2 Let $\varphi, \widetilde{\varphi} \in L^{2}(\mathbb{R})$ be as in the general setup, with trigonometric polynomial masks $m_{0}, \widetilde{m}_{0}$, respectively. Let $m_{1}, \widetilde{m}_{1}$ be trigonometric polynomials, and define $\psi_{1}, \widetilde{\psi}_{1} \in L^{2}(\mathbb{R})$ by (1.2). Assume that the conditions (a), (b) and (c) in Lemma 2.5 for $m_{0}, \widetilde{m}_{0}, m_{1}, \widetilde{m}_{1}$ are satisfied. Then the following are equivalent:

(I) There exist 1-periodic trigonometric polynomials $m_{2}, \widetilde{m}_{2}$ such that

(i) $m_{2}(0)=\widetilde{m}_{2}(0)=0$;

(ii) the matrix-valued functions $M, \widetilde{M}$ in (1.3) with $n=2$ satisfy

$$
\widetilde{M}(\gamma)^{*} M(\gamma)=I, \gamma \in \mathbb{T}
$$

(II) $\widetilde{M}_{\alpha}(\gamma) \widetilde{M}_{\alpha}(\gamma+1 / 2)=\widetilde{M}_{\beta}(\gamma) \widetilde{M}_{\beta}(\gamma+1 / 2), \gamma \in \mathbb{T}$.

In the affirmative case, the multi-wavelet systems $\left\{D^{j} T_{k} \psi_{\ell}\right\}_{\ell=1,2 ; j, k \in \mathbb{Z}}$ and $\left\{D^{j} T_{k} \widetilde{\psi}_{\ell}\right\}_{\ell=1,2 ; j, k \in \mathbb{Z}}$, with $\psi_{2}, \widetilde{\psi}_{2}$ defined by (1.2), form dual frames for $L^{2}(\mathbb{R})$.

Proof. $\quad(\mathrm{I}) \Rightarrow(\mathrm{II})$ : This follows from (2.2) and (2.3):

$$
\begin{aligned}
\widetilde{M}_{\alpha}(\gamma) \widetilde{M}_{\alpha}(\gamma+1 / 2) & =\overline{m_{2}(\gamma)} \widetilde{m}_{2}(\gamma) \overline{m_{2}(\gamma+1 / 2)} \widetilde{m}_{2}(\gamma+1 / 2) \\
& =\widetilde{M}_{\beta}(\gamma) \widetilde{M}_{\beta}(\gamma+1 / 2)
\end{aligned}
$$

$(\mathrm{II}) \Rightarrow(\mathrm{I})$ : Let $\widetilde{M}_{\alpha}, \widetilde{M}_{\beta}$ be factorized as (3.3) for 1-periodic trigonometric polynomials $\Lambda_{\alpha}, \Lambda_{\beta}$. Then Lemma 2.5 (b) implies

$$
\Lambda_{\alpha}(\gamma) \Lambda_{\alpha}(\gamma+1 / 2)=\Lambda_{\beta}(\gamma) \Lambda_{\beta}(\gamma+1 / 2)
$$

Let $\Gamma$ be the common factor of $\Lambda_{\alpha}$ and $\Lambda_{\beta}$, that is,

$$
\Lambda_{\alpha}(\gamma)=\Gamma(\gamma) \Gamma_{\alpha}(\gamma), \Lambda_{\beta}(\gamma)=\Gamma(\gamma) \Gamma_{\beta}(\gamma)
$$


for some 1-periodic trigonometric polynomials $\Gamma_{\alpha}, \Gamma_{\beta}$ with no common factors. This together with (3.4) implies

$$
\Gamma_{\alpha}(\gamma) \Gamma_{\alpha}(\gamma+1 / 2)=\Gamma_{\beta}(\gamma) \Gamma_{\beta}(\gamma+1 / 2)
$$

Since $\Gamma_{\alpha}$ and $\Gamma_{\beta}$ have no common factor, we have

$$
\Gamma_{\beta}(\gamma)=\Gamma_{\alpha}(\gamma+1 / 2)
$$

up to constant. Define $m_{2}$ and $\widetilde{m}_{2}$ by

$$
m_{2}(\gamma):=e^{-\pi i \gamma} \sin (\pi \gamma) \overline{\Gamma(\gamma)}, \tilde{m}_{2}(\gamma):=e^{-\pi i \gamma} \sin (\pi \gamma) \Gamma_{\alpha}(\gamma)
$$

Then (i) is trivial. By (3.5) and (3.6), we have

$$
\overline{m_{2}(\gamma)} \widetilde{m}_{2}(\gamma)=\sin ^{2}(\pi \gamma) \Gamma(\gamma) \Gamma_{\alpha}(\gamma)=\widetilde{M}_{\alpha}(\gamma)
$$

and

$$
\overline{m_{2}(\gamma)} \widetilde{m}_{2}(\gamma+1 / 2)=-i \sin (\pi \gamma) \cos (\pi \gamma) \Gamma(\gamma) \Gamma_{\alpha}(\gamma+1 / 2)=\widetilde{M}_{\beta}(\gamma)
$$

These lead to (ii) by (2.2) and (2.3) with $n=2$.

For the case where $\varphi=\widetilde{\varphi}=B_{2}$, we can characterize the possible trigonometric polynomials $m_{1}, \widetilde{m_{1}}$ with at most three terms that satisfy the conditions in Theorem 3.2 . Our main reason for stating this is that we can use the result to identify concrete candidates for pairs of wavelet systems that can not be extended to a pair of dual wavelet frames by adding a single pair of wavelet systems, see Example 3.5.

Corollary 3.3 Let $d_{0}, d_{1}, \widetilde{d}_{0}, \widetilde{d}_{1} \in \mathbb{C}$. Define $\psi_{1}$ and $\widetilde{\psi}_{1}$ by

$$
\begin{aligned}
& \psi_{1}(x):=d_{0} B_{2}(2 x)+\left(d_{1}-d_{0}\right) B_{2}(2 x-1)-d_{1} B_{2}(2 x-2) \\
& \widetilde{\psi}_{1}(x):=\widetilde{d}_{0} B_{2}(2 x)+\left(\widetilde{d}_{1}-\widetilde{d}_{0}\right) B_{2}(2 x-1)-\widetilde{d}_{1} B_{2}(2 x-2) .
\end{aligned}
$$

Then the following are equivalent:

(a) There exist 1-periodic trigonometric polynomials $m_{2}$ and $\widetilde{m}_{2}$ such that (I) in Theorem 3.2 holds;

(b) $3 \bar{d}_{0} \widetilde{d}_{0}+3 \bar{d}_{1} \widetilde{d}_{1}-\bar{d}_{1} \widetilde{d}_{0}-\bar{d}_{0} \widetilde{d}_{1}=2$.

Proof. Let $\varphi:=\widetilde{\varphi}:=B_{2}$ with the associated masks $m_{0}(\gamma):=\widetilde{m}_{0}(\gamma):=\left(e^{-\pi i \gamma} \cos (\pi \gamma)\right)^{2}$. From (3.7) and (3.8), $m_{1}$ and $\widetilde{m}_{1}$ are defined by

$$
m_{1}(\gamma)=\frac{d_{0}}{2}+\frac{d_{1}-d_{0}}{2} e^{-2 \pi i \gamma}-\frac{d_{1}}{2} e^{-4 \pi i \gamma}, \quad \widetilde{m}_{1}(\gamma)=\frac{\widetilde{d}_{0}}{2}+\frac{\widetilde{d}_{1}-\tilde{d}_{0}}{2} e^{-2 \pi i \gamma}-\frac{\widetilde{d}_{1}}{2} e^{-4 \pi i \gamma}
$$


Trivially, (a), (b) and (c) in Lemma 2.5 for $m_{0}, \widetilde{m}_{0}, m_{1}, \widetilde{m}_{1}$ are satisfied. We now check the condition (II) in Theorem 3.2. Note that

$$
\widetilde{M}_{\alpha}(\gamma)=1-\cos ^{4}(\pi \gamma)-\overline{m_{1}(\gamma)} \widetilde{m}_{1}(\gamma), \quad \widetilde{M}_{\beta}(\gamma)=\cos ^{2}(\pi \gamma) \sin ^{2}(\pi \gamma)-\overline{m_{1}(\gamma)} \widetilde{m}_{1}(\gamma+1 / 2)
$$

Then we have

$$
\begin{aligned}
\widetilde{M}_{\alpha}(\gamma) \widetilde{M}_{\alpha}(\gamma+1 / 2)= & \left(1-\cos ^{4}(\pi \gamma)\right)\left(1-\sin ^{4}(\pi \gamma)\right)-\left(1-\cos ^{4}(\pi \gamma)\right) \overline{m_{1}(\gamma+1 / 2)} \widetilde{m}_{1}(\gamma+1 / 2) \\
& -\left(1-\sin ^{4}(\pi \gamma)\right) \overline{m_{1}(\gamma)} \widetilde{m}_{1}(\gamma)+\overline{m_{1}(\gamma) m_{1}(\gamma+1 / 2)} \widetilde{m}_{1}(\gamma) \widetilde{m}_{1}(\gamma+1 / 2)
\end{aligned}
$$

and

$$
\begin{aligned}
& \widetilde{M}_{\beta}(\gamma) \widetilde{M}_{\beta}(\gamma+1 / 2) \\
& =\cos ^{4}(\pi \gamma) \sin ^{4}(\pi \gamma)-\sin ^{2}(\pi \gamma) \cos ^{2}(\pi \gamma) \overline{m_{1}(\gamma+1 / 2)} \widetilde{m}_{1}(\gamma) \\
& -\cos ^{2}(\pi \gamma) \sin ^{2}(\pi \gamma) \overline{m_{1}(\gamma)} \tilde{m}_{1}(\gamma+1 / 2)+\overline{m_{1}(\gamma) m_{1}(\gamma+1 / 2)} \tilde{m}_{1}(\gamma) \tilde{m}_{1}(\gamma+1 / 2) \text {. }
\end{aligned}
$$

Using the identities

$$
\begin{aligned}
& 1-\cos ^{4}(\pi \gamma)=\sin ^{2}(\pi \gamma)\left(1+\cos ^{2}(\pi \gamma)\right), \quad 1-\sin ^{4}(\pi \gamma)=\cos ^{2}(\pi \gamma)\left(1+\sin ^{2}(\pi \gamma)\right) \\
& \cos ^{2}(\pi \gamma)-\cos ^{4}(\pi \gamma)=\sin ^{2}(\pi \gamma)-\sin ^{4}(\pi \gamma)=\cos ^{2}(\pi \gamma) \sin ^{2}(\pi \gamma)
\end{aligned}
$$

the condition (II) in Theorem 3.2 is equivalent to

$$
\begin{aligned}
& \sin ^{2}(\pi \gamma) \cos ^{2}(\pi \gamma)\left(2-\left(\overline{m_{1}(\gamma)}-\overline{m_{1}(\gamma+1 / 2)}\right)\left(\widetilde{m}_{1}(\gamma)-\widetilde{m}_{1}(\gamma+1 / 2)\right)\right) \\
& =\sin ^{2}(\pi \gamma) \overline{m_{1}(\gamma+1 / 2)} \widetilde{m}_{1}(\gamma+1 / 2)+\cos ^{2}(\pi \gamma) \overline{m_{1}(\gamma)} \widetilde{m}_{1}(\gamma) .
\end{aligned}
$$

From (3.9), $m_{1}$ and $\widetilde{m}_{1}$ can be factorized as

$$
m_{1}(\gamma)=e^{-\pi i \gamma} \sin (\pi \gamma) \Lambda_{1}(\gamma), \quad \widetilde{m}_{1}(\gamma)=e^{-\pi i \gamma} \sin (\pi \gamma) \widetilde{\Lambda}_{1}(\gamma)
$$

where

$$
\Lambda_{1}(\gamma):=i\left(d_{0}+d_{1} e^{-2 \pi i \gamma}\right), \quad \widetilde{\Lambda}_{1}(\gamma):=i\left(\widetilde{d}_{0}+\widetilde{d}_{1} e^{-2 \pi i \gamma}\right) .
$$

Since $\overline{m_{1}(\gamma)} \widetilde{m}_{1}(\gamma)=\sin ^{2}(\pi \gamma) \overline{\Lambda_{1}(\gamma)} \widetilde{\Lambda}_{1}(\gamma)$, (3.10) is equivalent with

$$
2-\left(\overline{m_{1}(\gamma)}-\overline{m_{1}(\gamma+1 / 2)}\right)\left(\widetilde{m}_{1}(\gamma)-\widetilde{m}_{1}(\gamma+1 / 2)\right)=\overline{\Lambda_{1}(\gamma+1 / 2)} \widetilde{\Lambda}_{1}(\gamma+1 / 2)+\overline{\Lambda_{1}(\gamma)} \widetilde{\Lambda}_{1}(\gamma)
$$

This together with (3.9) and (3.11) leads to $2-\left(\bar{d}_{1}-\bar{d}_{0}\right)\left(\widetilde{d}_{1}-\widetilde{d}_{0}\right)=2\left(\bar{d}_{0} \widetilde{d}_{0}+\bar{d}_{1} \widetilde{d}_{1}\right)$, that is, $3 \bar{d}_{0} \widetilde{d}_{0}+3 \bar{d}_{1} \widetilde{d}_{1}-\bar{d}_{1} \widetilde{d}_{0}-\bar{d}_{0} \widetilde{d}_{1}=2$, as desired.

Let us consider a concrete case and find the functions $\psi_{2}, \widetilde{\psi_{2}}$ explicitly: 
Example 3.4 Let $d_{0}=1, d_{1}=0, \widetilde{d}_{0}=\frac{1}{2}, \widetilde{d}_{1}=-\frac{1}{2}$ and consider

$$
\psi_{1}(x)=B_{2}(2 x)-B_{2}(2 x-1), \widetilde{\psi}_{1}(x)=\frac{1}{2} B_{2}(2 x)-B_{2}(2 x-1)+\frac{1}{2} B_{2}(2 x-1),
$$

with the associated masks

$$
m_{1}(\gamma)=i e^{-\pi i \gamma} \sin (\pi \gamma), \quad \tilde{m}_{1}(\gamma)=-e^{-2 \pi i \gamma} \sin ^{2}(\pi \gamma)
$$

Then (b) in Corollary 3.3 holds. We now use (II) $\Rightarrow$ (I) in Theorem 3.2 to construct $\psi_{2}$ and $\tilde{\psi}_{2}$ such that the multi-wavelet systems $\left\{D^{j} T_{k} \psi_{\ell}\right\}_{\ell=1,2 ; j, k \in \mathbb{Z}}$ and $\left\{D^{j} T_{k} \widetilde{\psi}_{\ell}\right\}_{\ell=1,2 ; j, k \in \mathbb{Z}}$ form dual frames for $L^{2}(\mathbb{R})$. A direct calculation shows that

$$
\widetilde{M}_{\alpha}(\gamma)=\sin ^{2}(\pi \gamma) \Lambda_{\alpha}(\gamma), \quad \widetilde{M}_{\beta}(\gamma)=-i \sin (\pi \gamma) \cos (\pi \gamma) \Lambda_{\beta}(\gamma)
$$

where

$$
\Lambda_{\alpha}(\gamma)=\left(1+e^{-2 \pi i \gamma}\right)\left(e^{2 \pi i \gamma}+3\right), \quad \Lambda_{\beta}(\gamma)=\left(1+e^{-2 \pi i \gamma}\right)\left(e^{2 \pi i \gamma}-3\right)
$$

Letting $\Gamma(\gamma)=-i\left(1+e^{-2 \pi i \gamma}\right)$ we have $\Lambda_{\alpha}(\gamma)=\Gamma(\gamma) \Gamma_{\alpha}(\gamma), \Lambda_{\beta}(\gamma)=\Gamma(\gamma) \Gamma_{\beta}(\gamma)$, where $\Gamma_{\alpha}(\gamma)=i\left(e^{2 \pi i \gamma}+3\right), \Gamma_{\beta}(\gamma)=i\left(e^{2 \pi i \gamma}-3\right)$. Let

$$
\begin{aligned}
& m_{2}(\gamma)=e^{-\pi i \gamma} \sin (\pi \gamma) \overline{\Gamma(\gamma)}=e^{-\pi i \gamma} \sin (\pi \gamma) i\left(e^{2 \pi i \gamma}+1\right), \\
& \widetilde{m}_{2}(\gamma)=e^{-\pi i \gamma} \sin (\pi \gamma) \Gamma_{\alpha}(\gamma)=e^{-\pi i \gamma} \sin (\pi \gamma) i\left(e^{2 \pi i \gamma}+3\right) .
\end{aligned}
$$

and define $\psi_{2}, \widetilde{\psi}_{2}$ by $(1.2)$, i.e.,

$$
\psi_{2}(x)=B_{2}(2 x+1)-B_{2}(2 x-1), \quad \widetilde{\psi}_{2}(x)=B_{2}(2 x+1)+2 B_{2}(2 x)-3 B_{2}(2 x-1) .
$$

By Theorem [3.2, $\left\{D^{j} T_{k} \psi_{\ell}\right\}_{\ell=1,2 ; j, k \in \mathbb{Z}}$ and $\left\{D^{j} T_{k} \widetilde{\psi}_{\ell}\right\}_{\ell=1,2 ; j, k \in \mathbb{Z}}$ form dual frames.

Remember that our main motivation is the question whether any pair of Bessel wavelet systems can be extended to a pair of dual wavelet frames by adding a single pair of wavelet systems. Based on Corollary 3.3 we can state a concrete candidate for a counter example:

Example 3.5 Let $d_{0}=\widetilde{d}_{0}=1, d_{1}=\widetilde{d}_{1}=0$ and consider

$$
\psi_{1}(x)=\widetilde{\psi}_{1}(x)=B_{2}(2 x)-B_{2}(2 x-1) .
$$

Then we have

$$
3 \bar{d}_{0} \widetilde{d}_{0}+3 \bar{d}_{1} \widetilde{d}_{1}-\bar{d}_{1} \widetilde{d}_{0}-\bar{d}_{0} \widetilde{d}_{1}=3 \neq 2
$$

By Corollary [3.3, there does not exist a pair of $\psi_{2}, \widetilde{\psi}_{2}$ such that $\widetilde{M}(\gamma)^{*} M(\gamma)=I$ with $n=2$. Thus the wavelet systems $\left\{D^{j} T_{k} \psi_{1}\right\}_{j, k \in \mathbb{Z}}$ and $\left\{D^{j} T_{k} \widetilde{\psi}_{1}\right\}_{j, k \in \mathbb{Z}}$ can not be extended to dual pairs of MRA-based wavelet frames $\left\{D^{j} T_{k} \psi_{\ell}\right\}_{\ell=1,2 ; j, k \in \mathbb{Z}},\left\{D^{j} T_{k} \widetilde{\psi}_{\ell}\right\}_{\ell=1,2 ; j, k \in \mathbb{Z}}$. On the other hand it is an open question whether the systems $\left\{D^{j} T_{k} \psi_{1}\right\}_{j, k \in \mathbb{Z}},\left\{D^{j} T_{k} \widetilde{\psi}_{1}\right\}_{j, k \in \mathbb{Z}}$ can be extended to dual wavelet pairs $\left\{D^{j} T_{k} \psi_{\ell}\right\}_{\ell=1,2 ; j, k \in \mathbb{Z}},\left\{D^{j} T_{k} \widetilde{\psi}_{\ell}\right\}_{\ell=1,2 ; j, k \in \mathbb{Z}}$ for which the functions $\psi_{2}, \widetilde{\psi_{2}}$ do not have the MRA-structure. 
The following example illustrates the flexibility of the MEP-approach compared with the UEP. In fact, we will consider masks $m_{0}$ and $m_{1}$ as in the general setup, with the property that the wavelet system $\left\{D^{j} T_{k} \psi_{1}\right\}_{j, k \in \mathbb{Z}}$ can not be extended to a tight wavelet frame $\left\{D^{j} T_{k} \psi_{\ell}\right\}_{\ell=1,2 ; j, k \in \mathbb{Z}}$ using the UEP. On the other hand, by considering a second pair of masks $\widetilde{m_{0}}, \widetilde{m_{1}}$, the wavelet systems $\left\{D^{j} T_{k} \psi_{1}\right\}_{j, k \in \mathbb{Z}}$ and $\left\{D^{j} T_{k} \widetilde{\psi_{1}}\right\}_{j, k \in \mathbb{Z}}$ can be extended

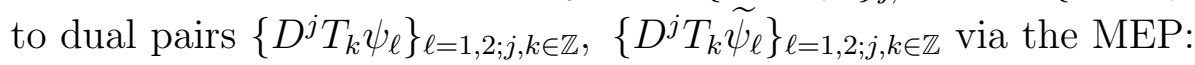

Example 3.6 Consider

$$
\varphi(x):=B_{1}(x), \quad \psi_{1}(x):=\frac{1}{4} B_{1}(2 x+2)+\frac{1}{4} B_{1}(2 x+1)-\frac{1}{4} B_{1}(2 x)-\frac{1}{4} B_{1}(2 x+2)
$$

with the associated masks

$$
m_{0}(\gamma)=e^{-\pi i \gamma} \cos (\pi \gamma), \quad m_{1}(\gamma)=i e^{\pi i \gamma} \sin (\pi \gamma) \cos ^{2}(\pi \gamma) .
$$

Let

$$
M_{\alpha}(\gamma)=1-\left|m_{0}(\gamma)\right|^{2}-\left|m_{1}(\gamma)\right|^{2}, \quad M_{\beta}(\gamma)=-m_{0}(\gamma) \bar{m}_{0}(\gamma+1 / 2)-m_{1}(\gamma) \bar{m}_{1}(\gamma+1 / 2) .
$$

A direct calculation shows that $M_{\alpha}(\gamma) M_{\alpha}(\gamma+1 / 2) \not \equiv M_{\beta}(\gamma) M_{\beta}(\gamma+1 / 2)$; by $[\underline{6}$, Theorem $2.2]$, this implies that there does not exist $m_{2} \in L^{\infty}(\mathbb{R})$ such that the multi-wavelet system $\left\{D^{j} T_{k} \psi_{\ell}\right\}_{\ell=1,2 ; j, k \in \mathbb{Z}}$, with $\psi_{2}$ defined by (1.2), forms a Parseval frame for $L^{2}(\mathbb{R})$.

On the other hand, consider now

$$
\widetilde{\varphi}(x):=B_{3}(x+1), \quad \widetilde{\psi}_{1}(x):=B_{3}(2 x+2)-B_{3}(2 x+1)
$$

with the associated masks $\widetilde{m}_{0}(\gamma)=e^{-\pi i \gamma} \cos ^{3}(\pi \gamma), \widetilde{m}_{1}(\gamma)=i e^{\pi i \gamma} \sin (\pi \gamma)$. A direct calculation shows that

$$
\widetilde{M}_{\alpha}(\gamma)=\sin ^{2}(\pi \gamma), \quad \widetilde{M}_{\beta}(\gamma)=-i \sin (\pi \gamma) \cos (\pi \gamma)
$$

Then we have

$$
\widetilde{M}_{\alpha}(\gamma) \widetilde{M}_{\alpha}(\gamma+1 / 2)=\widetilde{M}_{\beta}(\gamma) \widetilde{M}_{\beta}(\gamma+1 / 2)=\sin ^{2}(\pi \gamma) \cos ^{2}(\pi \gamma)
$$

It is easy to check that the conditions (a), (b) and (c) in Lemma 2.5 are satisfied, so by Theorem 3.2 there exists functions $\psi_{2}, \widetilde{\psi_{2}}$ such that $\left\{D^{j} T_{k} \psi_{\ell}\right\}_{\ell=1,2 ; j, k \in \mathbb{Z}}$ and $\left\{D^{j} T_{k} \widetilde{\psi}_{\ell}\right\}_{\ell=1,2 ; j, k \in \mathbb{Z}}$ form dual frames. By the proof of Theorem 3.2 we can define $\psi_{2}$ and $\widetilde{\psi}_{2}$ by

$$
\psi_{2}(x)=B_{1}(2 x)-B_{1}(2 x-1), \quad \widetilde{\psi}_{2}(x)=B_{3}(2 x+1)-B_{3}(2 x)
$$

with the associated masks $m_{2}(\gamma)=\widetilde{m}_{2}(\gamma)=i e^{-\pi i \gamma} \sin (\pi \gamma)$. 


\section{Extension with two pairs of generators}

For the sake of completeness we now prove that if the necessary conditions in Lemma 2.5]are satisfied, then we can always extend $\left\{D^{j} T_{k} \psi_{1}\right\}_{j, k \in \mathbb{Z}},\left\{D^{j} T_{k}{\widetilde{\psi_{1}}}_{\}_{j, k \in \mathbb{Z}}}\right.$ to dual wavelet frame $\left\{D^{j} T_{k} \psi_{\ell}\right\}_{\ell=1,2,3 ; j, k \in \mathbb{Z}},\left\{D^{j} T_{k} \widetilde{\psi}_{\ell}\right\}_{\ell=1,2,3 ; j, k \in \mathbb{Z}}$, by adding two pairs of wavelet systems with MRA-structure. Note that if it is not required that the functions $\psi_{2}, \psi_{3}, \widetilde{\psi_{2}}, \widetilde{\psi_{3}}$ have MRAstructure, it is trivial that such an extension exists: we can just take $\psi_{2}=\psi_{1}, \widetilde{\psi_{2}}=-\widetilde{\psi_{1}}$ to "cancel" the action of the given wavelet system, and then take $\psi_{3}$ and $\psi_{3}$ to be any pair of functions generating dual wavelet frames. But this argument does not work under the assumptions in the current paper. For example, if we consider the MRA-setup where the functions $\psi_{\ell}, \widetilde{\psi}_{\ell}$ are generated by a B-spline, $\varphi=B_{N}, N>1$, then there does not exist dual wavelet frames $\left\{D^{j} T_{k} \psi_{3}\right\}_{j, k \in \mathbb{Z}},\left\{D^{j} T_{k} \widetilde{\psi}_{3}\right\}_{j, k \in \mathbb{Z}}$ where

$$
\psi_{3}=\sum c_{k} B_{N}(2 x-k), \widetilde{\psi_{3}}=\sum \widetilde{c_{k}} B_{N}(2 x-k)
$$

with finite coefficient sequences, i.e., the masks $m_{3}, \widetilde{m_{3}}$ can not be trigonometric polynomials; see [9] (the result is repeated in Theorem 14.5.1 in [4]). Thus, we have to provide a different argument:

Theorem 4.1 Let $\varphi, \widetilde{\varphi} \in L^{2}(\mathbb{R})$ be as in the general setup, with trigonometric polynomial masks $m_{0}, \widetilde{m}_{0}$. Let $m_{1}, \widetilde{m}_{1}$ be trigonometric polynomials, and define $\psi_{1}, \widetilde{\psi}_{1}$ by (1.2). Assume that the conditions (a), (b) and (c) in Lemma 2.5 for $m_{0}, \widetilde{m}_{0}, m_{1}, \widetilde{m}_{1}$ are satisfied. Then there exist trigonometric polynomials $m_{2}, m_{3}, \widetilde{m}_{2}, \widetilde{m}_{3}$ such that $\left\{D^{j} T_{k} \psi_{\ell}\right\}_{\ell=1,2,3 ; j, k \in \mathbb{Z}}$ and $\left\{D^{j} T_{k} \widetilde{\psi}_{\ell}\right\}_{\ell=1,2,3 ; j, k \in \mathbb{Z}}$, with $\psi_{2}, \widetilde{\psi}_{2}, \psi_{3}, \widetilde{\psi}_{3}$ defined by (1.2), form dual frames for $L^{2}(\mathbb{R})$.

Proof. We construct $m_{2}, \widetilde{m}_{2}, m_{3}, \widetilde{m}_{3}$ such that

(1) $m_{2}(0)=\widetilde{m}_{2}(0)=m_{3}(0)=\widetilde{m}_{3}(0)=0$;

(2) the matrix-valued functions $M, \widetilde{M}$ in (1.3) with $n=3$ satisfy

$$
\widetilde{M}(\gamma)^{*} M(\gamma)=I, \gamma \in \mathbb{T}
$$

Choose $M_{\alpha}, M_{\beta}$ as in (3.1)-(3.2). We first define $m_{2}$ and $\widetilde{m}_{2}$ by

$$
\begin{aligned}
m_{2}(\gamma) & :=\overline{\widetilde{M}_{\alpha}(\gamma)}+\overline{\widetilde{M}_{\beta}(\gamma)}=\sin (\pi \gamma)\left(\sin (\pi \gamma) \overline{\Lambda_{\alpha}(\gamma)}+i \cos (\pi \gamma) \overline{\Lambda_{\beta}(\gamma)}\right) \\
\widetilde{m}_{2}(\gamma) & :=\sin ^{2}(\pi \gamma)
\end{aligned}
$$

where $\Lambda_{\alpha}$ and $\Lambda_{\beta}$ are defined as in (3.3). Then

$$
\begin{aligned}
1-\sum_{\ell=0}^{2} \overline{m_{\ell}(\gamma)} \widetilde{m}_{\ell}(\gamma) & =\widetilde{M}_{\alpha}(\gamma)-\overline{m_{2}(\gamma)} \widetilde{m}_{2}(\gamma)=\widetilde{M}_{\alpha}(\gamma)-\left(\widetilde{M}_{\alpha}(\gamma)+\widetilde{M}_{\beta}(\gamma)\right) \sin ^{2}(\pi \gamma) \\
& =\sin ^{2}(\pi \gamma) \cos (\pi \gamma)\left(\cos (\pi \gamma) \Lambda_{\alpha}(\gamma)+i \sin (\pi \gamma) \Lambda_{\beta}(\gamma)\right)
\end{aligned}
$$


and

$$
\begin{aligned}
\sum_{\ell=0}^{2} \overline{m_{\ell}(\gamma)} \widetilde{m}_{\ell}(\gamma+1 / 2) & =-\widetilde{M}_{\beta}(\gamma)+\overline{m_{2}(\gamma)} \widetilde{m}_{2}(\gamma+1 / 2) \\
& =\sin ^{2}(\pi \gamma) \cos (\pi \gamma)\left(\cos (\pi \gamma) \Lambda_{\alpha}(\gamma)+i \sin (\pi \gamma) \Lambda_{\beta}(\gamma)\right)
\end{aligned}
$$

We now define $m_{3}, \widetilde{m}_{3}$ by

$$
\begin{aligned}
& m_{3}(\gamma):=\sin (\pi \gamma)\left(\cos (\pi \gamma) \overline{\Lambda_{\alpha}(\gamma)}-i \sin (\pi \gamma) \overline{\Lambda_{\beta}(\gamma)}\right) \\
& \tilde{m}_{3}(\gamma):=\sin (\pi \gamma) \cos (\pi \gamma)
\end{aligned}
$$

Then it is easy to see that

$$
\begin{aligned}
& 1-\sum_{\ell=0}^{2} \overline{m_{\ell}(\gamma)} \widetilde{m}_{\ell}(\gamma)=\overline{m_{3}(\gamma)} \widetilde{m}_{3}(\gamma), \\
& \sum_{\ell=0}^{2} \overline{m_{\ell}(\gamma)} \widetilde{m}_{\ell}(\gamma+1 / 2)=-\overline{m_{3}(\gamma)} \widetilde{m}_{3}(\gamma+1 / 2),
\end{aligned}
$$

which is equivalent to the condition (2). By construction, (1) holds.

Let us illustrate Theorem 4.1 by returning to a construction presented in Example 2.5 in 6]. In that example, masks $m_{0}, m_{1}$ as in the general setup were considered, and it was shown how to extend the Bessel sequence $\left\{D^{j} T_{k} \psi_{1}\right\}_{j, k \in \mathbb{Z}}$ to a tight frame $\left\{D^{j} T_{k} \psi_{\ell}\right\}_{\ell=1,2,3 ; j, k \in \mathbb{Z}}$ using the UEP. However, the masks defining the functions $\psi_{2}, \psi_{3}$ are not trigonometric polynomials, so the functions $\psi_{2}, \psi_{3}$ are not compactly supported. Using the MEP we can now show that it is possible to extend $\left\{D^{j} T_{k} \psi_{1}\right\}_{j, k \in \mathbb{Z}}$ to a pair of dual wavelet frames with compactly supported generators. The example deals again with the B-splines, for convenience shifted to be centered around $x=0$ :

Example 4.2 Let $\ell \geq 2$. Consider

$$
\varphi(x):=B_{2 \ell}(x+\ell), \quad \psi_{1}(x):=\sum_{k=-\ell}^{\ell} \frac{1}{2^{2 \ell-1}}\left(\begin{array}{c}
2 \ell \\
\ell+k
\end{array}\right) B_{2 \ell}(2 x-k+\ell)
$$

with the associated masks

$$
m_{0}(\gamma)=\cos ^{2 \ell}(\pi \gamma), \quad m_{1}(\gamma)=\sin ^{2 \ell}(\pi \gamma)
$$

In [6, Example 2.5] it was shown that by letting

$$
\lambda_{1}(\gamma):=1-\left(\cos ^{2 \ell}(\pi \gamma)+\sin ^{2 \ell}(\pi \gamma)\right)^{2}, \lambda_{2}(\gamma):=1-\left(\cos ^{2 \ell}(\pi \gamma)-\sin ^{2 \ell}(\pi \gamma)\right)^{2},
$$

the masks $m_{2}, m_{3} \in L^{\infty}(\mathbb{T})$ defined by

$$
m_{2}(\gamma):= \begin{cases}\left(\sqrt{\lambda_{1}(\gamma)}+\sqrt{\lambda_{2}(\gamma)}\right) / 2, & \gamma \in[-1 / 2,0]+\mathbb{Z} \\ \left.\sqrt{\lambda_{1}(\gamma-1 / 2)}-\sqrt{\lambda_{2}(\gamma-1 / 2)}\right) / 2, & \gamma \in[0,1 / 2]+\mathbb{Z}\end{cases}
$$


and

$$
m_{3}(\gamma):=\left\{\begin{array}{ll}
\left(\sqrt{\lambda_{1}(\gamma)}-\sqrt{\lambda_{2}(\gamma)}\right) / 2, & \gamma \in[-1 / 2,0]+\mathbb{Z} \\
\left(\sqrt{\lambda_{1}(\gamma-1 / 2)}+\sqrt{\lambda_{2}(\gamma-1 / 2)}\right) / 2, & \gamma \in[0,1 / 2]+\mathbb{Z}
\end{array},\right.
$$

satisfy the UEP conditions. We note that these masks are not trigonometric polynomials.

Using the MEP we can now provide an alternative construction using trigonometric polynomials. Consider now $\widetilde{\varphi}:=\varphi, \widetilde{\psi}_{1}:=\psi_{1}$ with the associated masks

$$
\widetilde{m}_{0}(\gamma):=m_{0}(\gamma)=\cos ^{2 \ell}(\pi \gamma), \quad \widetilde{m}_{1}(\gamma):=m_{1}(\gamma)=\sin ^{2 \ell}(\pi \gamma)
$$

A direct calculation shows that

$$
\widetilde{M}_{\alpha}(\gamma)=\sin ^{2}(\pi \gamma) \Lambda_{\alpha}(\gamma), \widetilde{M}_{\beta}(\gamma)=-i \sin (\pi \gamma) \cos (\pi \gamma) \Lambda_{\beta}(\gamma)
$$

where

$$
\Lambda_{\alpha}(\gamma)=\left(\sum_{k=0}^{2 \ell-1} \cos ^{2 k}(\pi \gamma)\right)-\sin ^{4 \ell-2}(\pi \gamma), \quad \Lambda_{\beta}(\gamma)=-2 i \sin ^{2 \ell-1}(\pi \gamma) \cos ^{2 \ell-1}(\pi \gamma)
$$

Define trigonometric polynomials $m_{2}, \widetilde{m}_{2}, m_{3}, \widetilde{m}_{3}$ as in (4.1)-(4.4):

$$
\begin{aligned}
& m_{2}(\gamma):=\sin (\pi \gamma)\left(\sin (\pi \gamma) \overline{\Lambda_{\alpha}(\gamma)}+i \cos (\pi \gamma) \overline{\Lambda_{\beta}(\gamma)}\right), \quad \tilde{m}_{2}(\gamma):=\sin ^{2}(\pi \gamma) \\
& m_{3}(\gamma):=\sin (\pi \gamma)\left(\cos (\pi \gamma) \overline{\Lambda_{\alpha}(\gamma)}-i \sin (\pi \gamma) \overline{\Lambda_{\beta}(\gamma)}\right), \quad \tilde{m}_{3}(\gamma):=\sin (\pi \gamma) \cos (\pi \gamma)
\end{aligned}
$$

Then we see that the masks satisfy the conditions in Theorem $\underset{\sim}{4.1}$. Hence we conclude that $\left\{D^{j} T_{k} \psi_{\ell}\right\}_{\ell=1,2,3 ; j, k \in \mathbb{Z}}$ and $\left\{D^{j} T_{k} \widetilde{\psi}_{\ell}\right\}_{\ell=1,2,3 ; j, k \in \mathbb{Z}}$, with $\psi_{2}, \widetilde{\psi}_{2}, \psi_{3}, \widetilde{\psi}_{3}$ defined by (1.2), form dual frames for $L^{2}(\mathbb{R})$. By construction all the generators are compactly supported.

\section{References}

[1] L. Borup, R. Gribonval, M. Nielsen, Bi-framelet systems with few vanishing moments characterize Besov spaces, Appl. Comput. Harmon. Anal. 17 (2004) 3-28.

[2] M. Bownik, E. Weber, Affine frames, GMRA's, and the canonical dual, Studia Math. 159 (2003) 453-479.

[3] M. Charina, M. Putinar, C. Scheiderer, and J. Stöckler, A real algebra perspective on multivariate tight wavelet frames, Preprint, 2012.

[4] O. Christensen, An introduction to frames and Riesz bases, Birkhäuser 2003.

[5] O. Christensen, H. O. Kim, R. Y. Kim, Extensions of Bessel sequences to dual pairs of frames, Appl. Comput. Harmon. Anal. 34 (2013) 224-233. 
[6] O. Christensen, H. O. Kim, R. Y. Kim, On Parseval Wavelet Frames with Two or Three Generators via the Unitary Extension Principle, Canad. Math. Bull. to appear.

[7] C. Chui, Wavelets - a tutorial in theory and practice, Academic Press, San Diego, 1992.

[8] C. Chui, X. L. Shi, Inequalities of Littlewood-Paley type for frames and wavelets, SIAM J. Math. Anal. 24 (1993) 263-277.

[9] C. Chui, W. He, J. Stöckler, Compactly supported tight and sibling frames with maximum vanishing moments, Appl. Comput. Harmon. Anal. 13 (2002) 224-262.

[10] I. Daubechies, Ten Lectures on Wavelets, SIAM, Philadelphia, 1992.

[11] I. Daubechies, B. Han, Pairs of dual wavelet frames from any two refinable functions, Constr. Approx. 20 (2004) 325-352.

[12] I. Daubechies, B. Han, A. Ron, Z. Shen, Framelets: MRA-based constructions of wavelet frames, Appl. Comput. Harmon. Anal. 14 (2003) 1-46.

[13] M. Ehler, On multivariate compactly supported bi-frames, J. Fourier Anal. Appl. 13 (2007) 511-532.

[14] B. Han, Q. Mo, Splitting a matrix of Laurent polynomials with symmetry and its applications to symmetric framelet filter banks, SIAM J. Matrix Anal. Appl. 26 (2004) $97-124$.

[15] B. Han, Z. Shen, Dual wavelet frames and Riesz bases in Sobolev spaces, Constr. Approx. 29 (2009) 369-406.

[16] D. Han, Dilations and completions for Gabor systems, J. Fourier Anal. Appl. 15 (2009) 201-217.

[17] A. Petukhov, Symmetric framelets, Constr. Approx. 19 (2003) 309-328.

[18] A. Ron, Z. Shen, Affine systems in $L_{2}\left(\mathbb{R}^{d}\right)$ II: dual systems, J. Fourier Anal. Appl. 3 (1997) 617-637.

[19] A. Ron, Z. Shen, Compactly supported tight affine spline frames in $L_{2}\left(R^{d}\right)$, Math. Comp. 67 (1998) 191-207. 